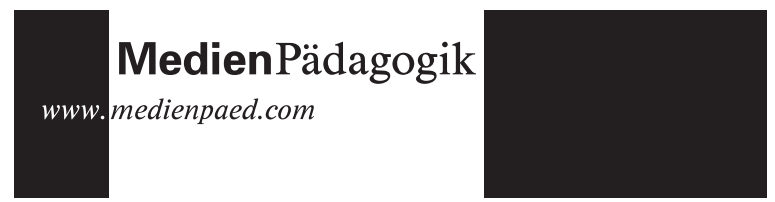

Theo Hug und Brigitte Hipfl

26.4.2006

\section{Medien-Gemeinschaften: \\ Aktuelle Diskurse und konzeptionelle Analysen}

In einer Zeit, in der bedingt durch die strukturellen Veränderungen in den Bereichen Familie und Arbeit, aber auch durch den Rückgang staatlicher Verantwortlichkeiten, traditionelle Formen der Zugehörigkeit immer mehr an Selbstverständlichkeit verlieren, schwindet jedoch nicht in gleicher Weise auch die Sehnsucht danach, irgendwo dazu zu gehören und nicht völlig auf sich allein gestellt zu sein. Wenn wir Steven Shaviros $(2003,64)$ Hinweis aufgreifen, dass heutzutage die Medien als Folie unseres Lebens fungieren, vergleichbar der Art und Weise, in der früher die «Natur» diese Funktion als Grundlage all unsere Sinnbezüge innegehabt hat, wird die Frage interessant, welche Möglichkeiten an Gemeinschaftserfahrungen Medien bereit stellen. Die neuen Informations- und Kommunikationstechnologien werben sogar ganz explizit mit den neuen Formen von Gemeinschaft, die sie bewirken und herstellen können. In diesem Beitrag setzten wir uns genauer mit dem Verhältnis von Medien und Gemeinschaft und entsprechenden Möglichkeiten der Modellierung auseinander. Wir machen dies aus mehreren Perspektiven und haben deshalb den Text in zwei Teile untergliedert. Im ersten Teil unternimmt Brigitte Hipfl einige Exkurse in verschiedene wissenschaftliche Disziplinen. Sie arbeitet den sozio-kulturellen Kontext für die Beziehung von Medien und Gemeinschaft heraus und zeigt auf, dass Medien eine lange Tradition in der Herstellung von Gemeinschaft haben und dass es sich bei diesem Thema um sehr grundlegende Fragen des menschlichen Zusammenlebens und des eigenen Selbstverständnisses handelt. Im zweiten Teil befasst sich Theo Hug mit einigen begrifflichen Aspekten der Thematik und der Pluralität an Konzepten und Verständnissen von Medien-Gemeinschaft. Er entwickelt ein Beispiel für eine Orientierungsmatrix zur Verortung spezifischer Diskursräume sowie insgesamt zehn Dimensionen, die im Zusammenhang von Charakterisierungen im Spannungsfeld von Medien und Gemeinschaften allesamt relevant sind. Diese Dimensionen werden hier nicht im Sinne einer Realdefinition, sondern als Perspektiven aufgefasst, mit denen verschiedene Beschreibungen von Medien und Gemeinschaft jeweils als Variationen sensu Nelson Goodman (1995) (re)konstruiert werden können.

\section{Gemeinschaft und Medien - eine sozio-kulturelle Verortung}

Gemeinschaft - das Versprechen von Sicherheit und Stabilität in einer immer unsicher werdenden Welt ${ }^{1}$

Es mag vielleicht auf den ersten Blick erstaunen, dass in jüngster Zeit gerade das Thema〈Gemeinschaft〉 wieder eine solche Konjunktur erlebt und bevorzugt Aspekte wie «community> und 〈community buildung〉 als positive Elemente der neuen Informations- und Kommunikationstechnologien angeführt werden. Eine Erklärung liefert Zygmunt Bauman (2001, 3f.), der darauf verweist, dass der Begriff Gemeinschaft mit all dem assoziiert wird, was uns gegenwärtig fehlt, wir aber glauben, dass wir es brauchen, um uns sicher zu fühlen. Bauman rekurriert in seinen Ausführungen auf das Alltagsverständnis von 〈Gemeinschaft», in dem davon ausgegangen wird, dass den Mitgliedern etwas gemeinsam ist, dass sie etwas mit anderen teilen. Dieses etwas kann die Sprache, Nationalität oder Religion sein, aber auch spezifische Interessen umfassen. Die Assoziationen beziehen sich darauf, mit anderen in einem durch gegenseitige Verantwortung, Reziprozität und emotionale Bindungen gekennzeichneten Verhältnis zu stehen und damit ein Gefühl des «Sich-zuhause-Fühlens» vermittelt zu bekommen. «Gemeinschaft» verspricht etwas, das sich gut anhört und als positiv eingeschätzt wird - im Gegensatz zu der Vorstellung, ganz alleine mit den Problemen des Alltags zurechtkommen zu müssen, diese mit niemandem teilen zu können, es mit unerwarteten und unbekannten Dingen zu tun zu haben, mit Menschen in Kontakt zu kommen, die man nicht kennt und bei denen man nicht sicher ist, dass man ihnen vertrauen kann. Demgegenüber steht Gemeinschaft für einen vertrauten, war-

Dieser Titel lehnt sich an den Untertitel von Zygmunt Baumans inspirierendem Buch «Community - Seeking Safety in an Insecure World» (2001) an. 
men und sicheren Ort, der von Menschen bevölkert ist, auf die man sich verlassen kann. Bauman weist jedoch darauf hin, dass dieses geradezu paradiesische Bild auch einen Preis verlangt: Freiheit und Autonomie müssen zugunsten der vom Kollektiv vermittelten Sicherheit aufgegeben werden. Wir haben es hier mit den Spannungsfeldern <emeinschaftIndividualität〉 bzw. 〈Sicherheit-Freiheit〉 zu tun, die für die menschliche Existenz konstituierend sind und nicht auf einfache Weise aufgelöst werden können. Unser Dilemma ist, dass wir beides, Sicherheit und Freiheit, benötigen und immer das Gefühl haben, nicht genug von beidem zu haben. Gerade unter den gegenwärtig bestimmenden Bedingungen des Neoliberalismus, in denen Individualismus, Konkurrenz (und Neid) im Mittelpunkt stehen, repräsentiert 〈Gemeinschaft〉 eine Welt, die uns nicht (mehr) zur Verfügung steht. Wir geben aber die Hoffnung nicht auf, dass wir neue Wege finden, die uns wieder gemeinschaftliche Erfahrungen ermöglichen. Natürlich beschäftigen sich auch Soziologen intensiv mit der Tatsache, dass sich traditionelle Formen von Gemeinschaft mehr und mehr auflösen. So konstatiert z. B. Ferdinand Tönnies in seinem 1878 erschienen Klassiker «Gemeinschaft und Gesellschaft» (Tönnis 1972) einen Wandel von 〈Gemeinschaft〉 zu〈Gesellschaft〉. Tönnies unterscheidet zwei historische Formen der Verbindung zwischen Menschen: die eine ist die Gemeinschaft, eine organische Form von Gemeinsamkeit, definiert durch ein bereits bestehendes gemeinsames Verständnis, das einfach da ist. Dies kann auf Blutszugehörigkeit (Familie, Grossfamilie) beruhen, aber auch bedingt sein durch das Leben an einem bestimmten Ort (durch die Nachbarschaft in einem Dorf, einer kleinen Stadt), oder auf einer mentalen Basis beruhen (wie z. B. Freundschaft). Was eine Gemeinschaft zusammenhält, ist die mit den anderen geteilte Art des Denkens, eine Art 〈Verstehen ohne Worte〉. Die andere Form ist die Gesellschaft, die keine gegebene, «natürliche〉 Einheit ist, sondern auf der Basis von Konventionen hergestellt wird. Hier werden bestimmte Aspekte von Gemeinsamkeit konstruiert und zur Grundlage des Handelns gemacht. Der von Tönnies beschriebene Wandel von Gemeinschaft zu Gesellschaft kann als sein Versuch gelesen werden, Industrialisierung und Modernisierung in ihrer Bedeutung für das menschliche Miteinander theoretisch zu fassen. Andere Soziologen (wie z. B. die beiden bekannten zeitgenössischen Vertreter Ulrich Beck (1995, 1996) und Anthony Giddens $(1996,2001)$ betonen ebenfalls die Auflösung traditioneller Bindungen und die damit verknüpften neuen Möglichkeiten, Herausforderungen und Ängste. Sie setzen sich auch mit der Frage aus- einander, welche neuen Formen von Gemeinschaften sich entwickeln werden und in welcher Weise diese für das Selbstverständnis ihrer Mitglieder von Bedeutung sind. Eine Gemeinschaft im Sinne von Tönnies kann nur solange existieren, solange sie nicht reflektiert wird, das heisst, solange das, was sie zusammenhält, nicht thematisiert und das intuitive, 〈heimliche〉 gemeinsame Verständnis nicht explizit gemacht wird (vgl. Bauman 2001, 11 f.). Demgegenüber charakterisiert Scott Lash (1996) die neuen Gemeinschaftsformen als «reflexive Gemeinschaften», in die die Menschen nicht mehr «geworfen werden», sondern in die sie sich selbst «hineinwerfen». Die Mitglieder sind sich der Konstruiertheit solcher Gemeinschaften und der Notwendigkeit, diese ständig selbst performativ herzustellen, bewusst. Das heisst unter anderem auch, dass die ästhetische Dimension einer Gemeinschaft eine grosse Rolle spielt: was als mit anderen geteilte Gemeinsamkeit wahrgenommen wird, beruht zu einem grossen Teil auf dem spezifischen Design einer Gemeinschaft und den expressiven Formen, in denen das Spezifische einer Gemeinschaft ausagiert wird. Konkret zeigt sich dies in verschiedenen Kleidungsstilen, Formen des Fantums am Beispiel von Populärkultur etc.

Anknüpfend an die soziologischen Diagnosen, die neben den veränderten Formen von Gemeinschaft besonders auf die radikale Individualisierung, die gegenwärtig stattfindet, verweisen, erfolgt in einem spezifischen Strang der politischen Philosophie, dem sog. Kommunitarismus, die Auseinandersetzung mit den ethischen Konsequenzen dieser Entwicklungen. Vertreter dieser Richtung (vgl. etwa den von Etzioni 1998 herausgegebenen Sammelband) fragen sich, welche Folgen dies für Demokratie, Zivilgesellschaft und die Wahrnehmung staatsbürgerlicher Rechte und Pflichten hat. Sie problematisieren die vom Neoliberalismus propagierte Individualisierung und die Tatsache, dass es uns nunmehr frei steht, zwischen verschiedenen Gemeinschaften, denen wir angehören können, wählen zu können. Und sie weisen darauf hin, dass damit auch die Orientierung auf ein gemeinsames Gut mehr und mehr in den Hintergrund gerät. Aber gerade letzteres ist ihnen wichtig und entsprechend weisen sie auch darauf hin, dass die Vorstellung von selbst bestimmten, autonomen Individuen, die mit individueller Anstrengung ihre Ziele erreichen können, etwas höchst Problematisches ist, da wir soziale Wesen - eingebettet in soziale Zusammenhänge - sind und deshalb auch Gemeinschaft so wichtig ist. Die Kritik wird sogar noch schärfer formuliert: Individualistische Positionen werden als parasitär dargestellt, bauen sie doch auf der Existenz von 
Gemeinschaften auf, untergraben diese aber gleichzeitig. Nach Etzioni $(1998, x)$ versuchen Kommunitaristen eine Balance zu finden zwischen den sozialen Kräften und den einzelnen Personen, zwischen Gemeinschaft und Autonomie, zwischen dem gemeinsamen Gut und individueller Freiheit, zwischen individuellen Rechten und sozialer Verantwortung. Kommunitaristen propagieren einen dritten Weg, nämlich Gemeinschaften, in denen bestimmte Pflichten definiert sind, die ihre Mitglieder zu erfüllen haben. Sie sehen dies als Alternative zur freien Marktwirtschaft auf der einen Seite und dem Staat auf der anderen Seite, und sie verfolgen die Vorstellung, damit die Zivilgesellschaft wieder zu beleben.

Aus einer kulturtheoretischen Perspektive wird zum einen mit dem Begriff der 〈kulturellen Identität〉 das Zusammenspiel von Gemeinschaft und Identität thematisiert (siehe etwa Hall 1994), zum anderen werden die Möglichkeiten, die die neue globale Mobilität und neue Migranten- und Transmigrantenkulturen eröffnen, erkundet (siehe etwa Robins/Aksoy 2001). Wenn hier von Identität gesprochen wird, dann soll in Anlehnung an Stuart Hall (1994, 188) veranschaulicht werden, wie Individualität gegenwärtig «gelebt, erfahren und begrifflich gefasst» wird. Es gilt inzwischen schon fast als Gemeinplatz, darauf hinzuweisen, dass 〈Identität〉 eben nicht das ist, was das Wort nahe legt, nämlich ein kohärentes, einheitliches Subjekt, das eine Art 〈Eins-Sein mit sich selbst〉 verkörpert. Vielmehr wird von Patchwork-Identitäten, fragmentierten und dezentrierten Identitäten gesprochen - Begriffe, die darauf verweisen, dass die strukturellen gesellschaftlichen Wandlungsprozesse, die mit solchen Schlagworten wie Globalisierung, Enttraditionalisierung, Hybridität, Transnationalität und Transkulturalität beschrieben werden, sich auch in unseren Selbstwahrnehmungen niederschlagen. Konkret heisst dies, dass mit Identität ein unser gesamtes Leben andauernder Prozess gemeint ist, in dem wir uns unter den spezifischen historischen Bedingungen, unter denen wir leben, mit einigen der uns zur Verfügung stehenden sozialen Kategorien (bzw. mit Menschen, die diese Kategorien repräsentieren) identifizieren. Diese werden zu Identitätspositionen, die über unterschiedlichen sozialen Status verfügen - manche sind sehr anerkannt und werden von vielen als erstrebenswert angesehen, andere wieder gelten aufgrund der vorherrschenden Machtrelationen als weniger attraktiv oder gar als negativ. Dies lässt sich am Beispiel von Medienstars auf der einen Seite und bestimmten ethnischen Gruppen, die in diversen kulturellen Kontexten als fremd und andersartig repräsentiert werden, auf der anderen Seite, sehr gut illustrieren. Diese sozialen Bedeutungen, die bestimmten sozialen Gruppen oder Gemeinschaften zugeschrieben werden, sind nicht deren Wesensmerkmale, sondern das Resultat von sozialen Praktiken, mit denen diese Bedeutungen ständig reproduziert werden. Hier haben wir es mit der performativen Dimension des sozialen Handelns zu tun - durch die Art und Weise, wie wir handeln und agieren, stellen wir immer wieder diese Bedeutungen (und damit Identitäten im Sinne von Zugehörigkeiten zu bestimmten Gemeinschaften) her. Gleichzeitig liegt das nicht nur an uns, wir können dies nicht alleine bestimmen, vielmehr kommt den Anderen eine zentrale Rolle dabei zu, ob sie uns in diesen Identitätspositionen und als Mitglied bestimmter Gemeinschaften akzeptieren. Hier wird deutlich, dass Gemeinschaften sensible und im höchsten Sinne politische Angelegenheiten sind, geht es doch um so wichtige Fragen wie: Wer kann einer Gemeinschaft angehören und wer nicht? Was gilt als notwendige Voraussetzung dafür, als Mitglied anerkannt zu werden? Welche Grenzziehungen werden zum Schutz einer Gemeinschaft vorgenommen, welche Massnahmen werden gesetzt, um sich von anderen Gemeinschaften zu differenzieren? Neben der Tatsache, dass Gemeinschaften ständig mit Entscheidungen über die Inklusion und Exklusion von Mitgliedern konfrontiert sind, stellt sich aber auch die Frage nach den Elementen, die Gemeinschaften zusammenhalten und definieren. Hier kommt es oft zu den Problemen, die mit dem Begriff der 〈Identitätspolitik〉 beschrieben werden und sich darin niederschlagen, dass ein Merkmal (wie etwa die $\mathrm{Zu}$ gehörigkeit zu einer ethnischen Gruppe oder die sexuelle Orientierung) als dasjenige angesehen wird, das alle teilen und aus dem sich gemeinsame Interessen ergeben. Dass aber auch Differenzen zwischen den Mitgliedern einer Gemeinschaft bestehen, kommt dabei zu kurz. Hier zeigt sich erneut das schon weiter vorne angesprochene grundsätzliche Problem bei Gemeinschaften: die Vorstellung, dass es sich dabei um homogene Gruppen handelt.

Wenden wir unseren Blick jedoch den Veränderungen zu, die die globale Mobilität (von Menschen, aber auch von Medien) mit sich bringt, dann stossen wir auf Formen von Zusammengehörigkeit, die als Diaspora bzw. als Gemeinschaften beschrieben werden, die sich in transnationalen und transkulturellen Zusammenhängen entwickeln. Ihnen ist gemeinsam, dass nicht länger eine bestimmte nationale Zugehörigkeit bestimmend dafür ist, wie sich Personen hinsichtlich ihrer kulturellen Identität verstehen, sondern die gleichzeitige Existenz mehrerer kultureller Kontexte, die für sie be- 
deutsam sind. Dieses Thema wird in letzter Zeit auch verstärkt filmisch bearbeitet, wie etwa am Beispiel von jungen Menschen mit einem türkischen Migrationshintergrund in Deutschland (vgl. den Film Gegen die Wand, 2003, Regie: Fatih Akin) oder Jugendliche, deren Familien aus Indien nach England eingewandert sind (wie in Bend it like Beckam, 2002, Regie: Gurinder Chadha). Dabei werden auch die Konflikte angerissen, die sich gerade für junge Menschen aus der Situation ergeben, dass sie einerseits vielfach von ihren Eltern angehalten werden, ihre (oft in nostalgischer Weise fixierte und <eingefrorene〉) Herkunftskultur aufrechtzuerhalten und zu pflegen, sie sich selbst aber nicht nur über diese, sondern auch über die kulturellen Bedeutungen des kulturellen Kontextes, in dem sie nun leben, definieren. Diese jungen Menschen bewegen sich in transnationalen und transkulturellen Räumen und verkörpern hybride Identitäten.

\section{Gemeinschaftsbildende Funktionen der Medien}

Die konstitutive Bedeutung, die die Medien für die Entwicklung von Gemeinschaften einnehmen, soll in diesem Abschnitt aufgezeigt werden. Das Zusammenspiel von Medien und Gemeinschaft ist kein so neues Thema, wie es uns aufgrund der Diskussionen um virtual communities, web communities etc. erscheinen mag. Denn Medien dienen nicht nur der Information und Unterhaltung, immer schon wurden sie auch zu Herrschaftszwecken eingesetzt und haben die Funktion erfüllt, ein Gefühl von Gemeinschaft und Zusammengehörigkeit zu erzeugen. So hat etwa der Kanadier Harold Innis (vgl. Innis, 1951, 1972) in seiner Geschichte der Medien die Rolle deutlich gemacht, die insbesondere den Medien für die Konstitution, Aufrechterhaltung, aber auch für die Veränderung gesellschaftlicher Formationen zukommt. Dies kann als ein Ansatz interpretiert werden, der einen Einblick in die Funktion vermittelt, die der medialen Infrastruktur für politische und sozio-kulturelle Einheiten zukommt. In Innis' Terminologie ist eine bestimmte Herrschaftsform davon abhängig, wie erfolgreich sie in der Beherrschung eines Raumes bzw. in der Herrschaft über die Zeit sind. Er entwickelte dementsprechend auch eine Systematisierung der Medien, die er in «leichte» und «schwere» unterteilt, je nachdem, ob sie besser dazu geeignet sind, Raum zu überwinden (wie im Falle von Schriftzeichen auf Papier und Papyrus) oder Zeit zu überdauern (wie etwa im Fall der Tontafeln).

Benedict Anderson (1988) hat die subtile Art und Weise, in der bestimmte Herrschaftsformen mithilfe von Medien funktionieren, mit dem Konzept der «imagined community», der «vorgestellten Gemeinschaft» beschrieben. Er verweist darauf, dass alle Gemeinschaften, die eine Grösse haben, bei der sich nicht mehr alle Mitglieder von Angesicht zu Angesicht kennen, «vorgestellte Gemeinschaften» sind. Dazu braucht es etwas, das diese Vorstellung einer zusammengehörenden Einheit bewirkt und aufrechterhält. Dies sind gegenwärtig die Medien, denen im Prinzip dieselbe Funktion zukommt, die zuvor von den grossen kulturellen Systemen wie Weltreligionen und Dynastien erfüllt wurden - nämlich als Bezugsrahmen für existenzielle menschliche Fragen zu fungieren. In den Religionen hat die «heilige Sprache» (wie Latein oder klassisches Arabisch), in der die göttliche Wahrheit zum Ausdruck kam, diese einheitsstiftende Funktion innegehabt. Die Briefe des Apostel Paulus, die in den Anfängen der römisch-katholischen Kirche in der Zeit zwischen 48 bis 60 nach Christus an die katholischen Gemeinden in Rom, Thessaloniki, Korinth etc. gerichtet waren, ${ }^{2}$ können als frühe Beispiele dessen gesehen werden, was heute als «community building» bezeichnet wird. Paulus hatte nur einige dieser Gemeinden besucht und kannte nicht alle Mitglieder. Aber dadurch, dass er sie als Mitglieder einer Glaubensgemeinschaft ansprach und in seinen Briefen katholische Grundannahmen, Überzeugungen und Wertvorstellungen als die normative Basis für die Auseinandersetzung mit lebenspraktischen Themen und Problemen präsentierte, trug er gleichzeitig dazu bei, dass sich die einzelnen Katholiken und Katholikinnen als einer Gemeinschaft zugehörig fühlten konnten, in der sie ihren Glauben mit den anderen teilen. Ähnlich funktionierte es in den grossen Dynastien, in denen die jeweiligen Herrscher als Fundament einer Gemeinschaft wirkten, indem sie die gültige Ordnung repräsentierten und über ihre Kundmachungen und diversen Vollzugsmassnahmen zur Anwendung brachten.

Andersons besonderer Verdienst ist, als einer der ersten auf die gemeinschaftsbildende Funktion massenproduzierter Medien hinzuweisen. Er beschreibt, wie die kapitalistischen Interessen an der Erfindung des Buckdrucks dazu führten, dass immer mehr Bücher in regionalen Sprachen auf den Markt gebracht wurden - und zwar nicht in den vielen verschiedenen Sprachen, die gesprochen wurden, sondern in einigen wenigen ausgewählten «Drucksprachen». Für Anderson ist dies eine wesentliche Voraussetzung für die Entwicklung von Nationalismus und Nationalstaaten zu Ende des 18. Jahrhunderts. Denn erst aufgrund der Tatsache, dass es nun

\footnotetext{
Vgl. «http://de.wikipedia.org/wiki/Apostel_Paulus〉
} 
erstmals den Sprecher/innen der unterschiedlichen Formen von Englisch oder Französisch möglich war, sich gegenseitig über Bücher und Zeitungen $\mathrm{zu}$ verständigen und sich die Leser/innen dieser Produkte damit bewusst wurden, dass es noch Tausende Menschen gibt, die ihrer Sprache angehören und sie mit ihnen durch und über diese Printmedien verbunden sind, entwickelte sich so etwas wie die Vorstellung eines nationalen Bewusstseins. Wie die Inhalte etwa in Romanen oder in Zeitungen dargestellt wurden, spielt dabei eine nicht unwesentliche Rolle. Anderson charakterisiert dies als die Konstruktion einer «nationalen Imagination>, in der den Leser/innen eine stabile, sich durch die Zeit bewegende Gemeinschaft vorgeführt wird, der sie sich selbst durch das Lesen zugehörig fühlen. Dieses Muster wird uns immer noch sehr anschaulich von den nationalen Rundfunk- und Fernsehanstalten vorgeführt. Dort werden wir etwa in den Informationssendungen als Angehörige einer nationalen Einheit angesprochen, mit den Inhalten wird in subtiler und banaler Weise (vgl. Billig, 1995) das Gemeinsame in immer wiederkehrenden Wiederholungen dargestellt oder genauer gesagt, konstruiert. Beispiele dafür sind etwa solch unbedeutend erscheinende Worte wie «wir» oder «uns», mit denen wir als Angehörige einer Gemeinschaft adressiert werden, oder die Wettervorhersagen, in denen immer die national-geografischen Spezifika im Mittelpunkt stehen und überhaupt die ständige Wiederkehr von Vertrautem (seien dies nun bestimmte Formate, Bilder oder Sprecher/innen). Das sind die Mechanismen, mit denen, folgen wir John Corner (2001, 263), nicht nur das offizielle Leben einer Nation konstruiert wird, sondern auch die privaten, populären Formen des Vergnügens. All dies beschreibt Orvar Lofgren (zit. in Morley, 2001, 21) als «Mikrophysik des Lernprozesses der Zugehörigkeit» zu einer Nation als Zuhause», die den Effekt hat, dass sich die Menschen in einer Nation heimisch fühlen. Eigentlich ist dies, so Lofgren (zit. in Morley, 2001, 23) eine «Nationalisierung» des häuslichen Lebens und eine «Domestizierung» des Nationalen. Auf diese Weise kommt es zur Vorstellung einer nationalen Gemeinschaft, die sich von anderen Gemeinschaften abgrenzt, deren Mitglieder sich untereinander nicht face-to-face kennen und die trotz der sozialen Unterschiede, die zwischen den einzelnen Mitgliedern bestehen, von einer sie verbindenden Gemeinsamkeit ausgehen.

David Morley (2001) weist jedoch eindrücklich darauf hin, dass die Art und Weise, von wem und wie die Medieninhalte repräsentiert werden, schwerwiegende Konsequenzen im Hinblick darauf nach sich zieht, wer sich durch diese Form der öffentlichen Zirkulation von Bildern dessen, was als typisch und charakteristisch für eine nationale Gemeinschaft gilt, angesprochen und infolgedessen auch dazugehörig fühlt. Seine Diagnose für das britische Fernsehen ist ernüchternd: obwohl Grossbritannien längst eine multikulturelle Gesellschaft ist, dominiert in Rundfunk und Fernsehen eine «weisse» Perspektive. Das heisst, es werden in erster Linie die kulturellen Praktiken und die Anliegen der weissen angelsächsischen Bevölkerung ausgestrahlt. Wer in diesen Repräsentationen nationaler Normalität nicht oder wenn, dann nur als (vielfach auch negativ konnotiertes) Anderes vorkommt, fühlt sich entsprechend auch nicht einbezogen in diese Form kollektiver Identität. Morley zitiert mehrere Studien an Angehörige ethnischer Minderheiten, in denen ihre Unzufriedenheit mit dem britischen Fernsehen, das zu ihren Themen und Fragen praktisch nichts anbietet, sowie ihr grosses Interesse an Video-Kabel- und Satellitenfernsehen, in deren Angeboten sie sich viel stärker wieder finden, zum Ausdruck kommt. An diesem Beispiel wird die ambivalente Situation, in der sich gegenwärtig auch die Medieninstitutionen finden, deutlich: Einerseits zeigt sich, welchen neuen Herausforderungen sich nationale Rundfunk- und Fernsehanstalten zu stellen haben, wollen sie tatsächlich zur Entwicklung eines Gefühls von Gemeinschaft beitragen, das der Vielfältigkeit und den Unterschieden, wie sie in der multikulturellen Gesellschaft existieren, gerecht wird. Andererseits sehen wir die Effekte des Globalisierungsprozesses am Beispiel der weltweiten Zirkulation von Medieninhalten und der durch Kabel- und Satellitentechnologien zunehmend ortsunabhängigen Zugriffsmöglichkeit zu Medieninhalten, was insgesamt zu einer Unterminierung der nationalen Medienangebote führt. Entsprechend bewegen sich gerade Angehörigen ethnischer Gruppen immer mehr in so genannten transnationalen Räumen, da sie gleichzeitig Medien aus verschiedenen nationalen Kontexten (aus ihrem Herkunftsland, aus dem Land, in dem sie sich gerade aufhalten, aber auch aus anderen Ländern) rezipieren können. In theoretischer Hinsicht besteht hier, wie z. B. Kevin Robins immer wieder vehement beklagt, die Gefahr, sich bei den konzeptionellen Erklärungen dieser Phänomene zu sehr von der «hartnäckige(n), beharrliche(n) Idee der (nationalen) 〈vorgestellten Gemeinschaft〉» (Robins/Aksoy 2001, 74) leiten zu lassen und mit der Fokussierung auf die Zugehörigkeit zu verschiedenen Gemeinschaften gerade das Neue und Besondere an transkulturellen Erfahrungen zu übergehen. Hier wäre nach Robins/Aksoy $(2001,107)$ eine kosmopolitsche Perspektive angemessen, die die «Schaffung alternativer 
Arten kultureller Vereinbarungen» anstrebt und dem gegenseitigen Austausch und den Übersetzungsakten zwischen den Kulturen eine zentrale Rolle einräumt.

Ein zweiter Diskussionsstrang zum Zusammenhang von Medien und Gemeinschaft konzentriert sich auf die neuen Informations- und Kommunikationstechnologien. Howard Rheingold (1994) war einer der ersten Proponenten von 〈virtuellen Gemeinschaften〉, in denen sich Menschen mit gleichen Interessen, Problemen etc. im Internet finden und sich gegenseitig stützen. Voll Enthusiasmus spricht er von der ermächtigenden Wirkung, die die weltweiten selbst-bestimmten Zusammenschlüsse von Menschen im Netz mit sich bringen. Ähnlich betonen auch Vertreter/innen marginalisierter Gruppen das demokratisierende Potenzial von cyber-communities. Pierre Lévy (1997) geht noch einen Schritt weiter und skizziert am Beispiel der computer-basierten Technologien wie Internet oder World Wide Web die Utopie einer neuen Form menschlicher Kultur, die er als «kollektive Intelligenz> bezeichnet. Gemeint ist damit die Konstruktion intelligenter Gemeinschaften, in denen aufgrund eines Wechsels von singulärem zu pluralem Denken (also von 〈cogito - ich denke〉 zu 〈cogitamus - wir denken>) unser soziales und kognitives Potenzial gemeinsam weiter entwickelt werden kann (vgl. Levy 1997, 20 f.). Neben diesen optimistischen Einschätzungen der Möglichkeiten der so genannten neuen Medien gibt es aber auch viele kritische Stimmen zu virtuellen Gemeinschaften. Stellvertretend zitiere ich Steven Shaviro (2003, 31 f.), der etwa im Gegensatz zu Vorstellungen eines freien und demokratischen Cyberspace darauf hinweist, dass den (häufig als Netzwerke charakterisierten) medialen Bindungen die Mechanismen sozialer Kontrolle gewissermassen inhärent sind und gar nicht mehr von aussen aufgesetzt werden müssen.

\section{Medien-Gemeinschaft:}

\section{Dimensionen und konzeptionelle Gesichtspunkte}

Diese Skizze relevanter Diskursstränge macht deutlich, dass wir es mit einer Vielfalt an Konzepten und Verständnissen zu tun haben, die jeweils unterschiedliche Ebenen und Perspektiven in den Vordergrund rücken. Dabei fällt auf, dass auch im Deutschen der Ausdruck «Communities» häufiger anzutreffen ist als der Ausdruck «Gemeinschaften». Dies dürfte nicht nur mit der missbräuchlichen Vereinnahmung des Gemeinschaftsbegriffs während der Zeit des Nationalsozialismus oder mit der zunehmenden Verbreitung von Anglizismen, sondern auch mit der Popularisierung von sprachlichen Wendungen zusammenhängen, die in alltagspraktischen, technologischen, politischen und wissenschaftlichen Diskurskontexten figurieren oder zumindest anschlussfähig sind. Der Preis für die «Drehtür»Funktion, die mit Ausdrücken wie «Online-Community» oder «virtuelle Gemeinschaft» einhergeht, ist freilich eine gewisse Verflachung der Diskussion. Wenn beispielsweise von «Web-Didaktik als Teil der E-LearningDidaktik», von «Flash-Content, der ins Netz gestellt wurde» oder von «Communities, die angemailt wurden» die Rede ist, dann haben wir es in aller Regel mit praktischen Aspekten der Medien- und Bildungstechnologie zu tun, die unter theoretischen Gesichtspunkten hochgradig explikationsbedürftig sind.

Die Gründe für den hohen Verbreitungsgrad solcher oberflächlichen Begriffsverwendungen sind vielfältig. Sie reichen von mikropolitischen Formen der Identitätsdarstellung über marktbezogene Vereinfachungen, technologische Dynamiken und diskursstrategische Einsätze bis hin zur Relevanz der globalisierten Finanzmärkte. Andererseits lässt sich schon ohne umsichtige Begriffsanalysen zeigen, dass wir es hier mit sehr unterschiedlichen Bedeutungsfeldern zu tun haben. Aspekte wie Nachbarschaftshilfe oder Gemeinwesen haben im Zusammenhang des englischen Begriffs 〈Community〉 gemeinhin eine grosse Bedeutung, während sie mit Blick auf den deutschen Begriff 〈Gemeinschaft〉 nur in Spezialdiskursen vorkommen. Ähnlich verhält es sich mit der Verknüpfung der Schlüsselbegriffe 〈Community〉 und 〈Media〉. Optische, akustische und begriffliche Elemente der beiden Ausdrücke können hier bereits durch ihre Anordnung Spannungsfelder erzeugen, die nach einer Klärung des Verhältnisses der beiden Ausdrücke verlangen. So bezieht sich «Community Media» typischerweise auf lokale Medieninstitutionen oder regionale Programmangebote, während «Media-Community» häufig als Sammelbegriff für Mediengemeinschaften auf der Basis spezifischer Interessen und Nutzungsformen bestimmter Technologien verwendet wird. «Media \& Community» meint dagegen die Mannigfaltigkeit der Verknüpfungsmöglichkeiten diverser Bedeutungshorizonte von Medien und Gemeinschaft. Solche Verknüpfungen sind unterschiedlich verbreitet und sie können im Rahmen kultureller Symboliken und gesellschaftlicher Kontexte jeweils in verschiedener Weise sinnhaft getätigt werden. Im Prinzip kommen dabei diverse Kombinationen der unterschiedlichsten Verständnisse der Ausgangsbegriffe in Betracht. Wir wollen hier wenigstens exempla- 
risch andeuten, welche Aspekte der Differenzierung in diesem Zusammenhang in Betracht gezogen werden können.

In der nachfolgenden Tabelle greifen wir dazu auf einige Grundverständnisse der Begriffe〈Gemeinschaft〉 und 〈Medien〉 zurück. So lassen sich grosso modo Primärgruppen, die sich durch relativ stabile, intime und vertrauensvolle Beziehungen auszeichnen, unterscheiden von bereichsspezifischen Gemeinschaften mit unterschiedlichen Reichweiten, ethnischen Gemeinschaften sowie Gemeinwesen und «Communities» mit Akzentsetzungen, die von «Social Work» bis «e-Commerce» reichen. Ähnlich wählen wir aus der Vielfalt möglicher Unterscheidungen von Medienbegriffen eine kleine Auswahl. Hier bietet sich an die Hilfsmittel der Verständigung, die Kommunikationsmittel, von Medienangeboten sowie Instrumenten, Geräten, Apparaten und Medienorganisationen zu unterscheiden (vgl. Schmidt 1994, 83). Zwecks Kontrastierung nehmen wir zusätzlich zwei weitere Medienbegriffe auf, die im Kontext soziologischer Theoriebildung bedeutsam geworden sind: symbolisch generalisierte Kommunikationsmedien und die Unterscheidung Medium / Form (vgl. Luhmann 1987, 222; 1995, 168).

Die einzelnen Begriffe der Zeilen und Spalten in der Tabelle spannen jeweils spezifische Diskursräume auf, die bestimmte Arten und Weisen des Sprechens und Reflektierens über Mediengemeinschaften eröffnen. Die Einträge in den Feldern der Tabelle haben wie die Begriffswahl selbst allesamt exemplarischen Charakter. Sie stellen Beispiele für mögliche Knotenpunkte im Netz der diskursiven Möglichkeiten dar und zeigen, dass die Rede von Mediengemeinschaften bei näherer Betrachtung hochgradig differenziert werden kann. Dies lässt sich an beliebigen Beispielen leicht aufzeigen. Nehmen wir etwa den Ausdruck «Web-Community». Wenn wir davon ausgehen, dass die Elemente eines Mediums lose gekoppelt sind und dass die Form eine mehr oder weniger dauerhafte, feste Kopplung desselben Typs von Elementen darstellt, dann lässt die Rede von der «Web-Community» wie folgt paraphrasieren: Ähnlich wie im Medium der Buchstaben die Wörter Formen oder wie im Medium der Wörter die Sätze Formen darstellen können, kann im Medium aller Medien-Nutzer und -Nutzerinnen die Nutzung des World Wide Web (WWW) im Sinne einer relativ festen Kopplung mittels Hypermedia-Technologien als «Web-Community» beschrieben werden. Fanzines von und für Fussballclubs hingegen sind für die Konstitution von Gemeinschaften in anderer Weise bedeutsam als Blasmusikkapellen in ländlichen Gegenden oder Radio-Sender wie
EthnoTechno.com, die sich selbst als «new voice from the concrete jungles of this planet, delivering the music of a futuristic world peopled by the modern and ancient alike. ETHNIC-ally rich, TECHNO-logically enhanced, a new groove for a groovin $>$ new world $»^{3}$ verstehen. In allen diesen Fällen sind unterschiedliche Medien- und Gemeinschaftsverständnisse mit diversen Akzentuierungen sozialer, kultureller, politischer, wirtschaftlicher und technologischer Dimensionen konstitutiv.

Angesichts der Vielfalt einschlägiger Begriffe und Konzeptionen lassen sich hier allenfalls spezifische Akzente und prototypische Beispiele hervorheben. Hinzu kommt, dass eine Matrix etwa mit den Begriffen «Gruppe», «Gemeinschaft» und «Team» und einer Differenzierung von Medien entlang technischer Kriterien wie Speicher- und Übertragungsmedien andere Beispiele enthalten und andere Diskussionshorizonte eröffnen würde.

Analoges gilt für «Virtuelle Gemeinschaften». Auch hier lassen sich Virtualitätsbegriffe differenzieren. Die heute verbreiteten Auffassungen von «virtuell» im Sinne von «auf Digitalisierungsprozessen beruhend» haben nur indirekt mit traditionellen Verständnissen von «der Kraft oder Möglichkeit nach vorhanden» zu tun. ${ }^{4}$ Der Rückgriff auf eine verbreitete Untergliederung von Medien in primäre Medien (Bsp. Gespräch ohne technische Hilfsmittel), sekundäre Medien (nur einer der Partner benutzt technische Hilfsmittel, Bsp. Printmedien), tertiäre Medien (Kommunikator und Rezipient benutzen technische Hilfsmittel, Bsp. Radio, Fernsehen) und quartäre Medien (computerbasierte Kommunikation, Bsp. Internet) macht schnell deutlich, dass «Virtual Communties» meist synonym mit «OnlineCommunities» aufgefasst werden. Die Reflexion alternativer Möglichkeiten bleibt meist ausgeklammert. Sowohl die Gruppenpädagogik als auch die Medienwissenschaften bieten allerdings weit mehr Potenziale der Differenzierung als verbreitete Redeweisen von «virtuellen Gemeinschaften» uns weismachen wollen.

3 Vgl. das Broadcaster Profile unter

http://www.live365.com/stations/dimmsummer>

${ }^{4}$ Leider wird auch in der Medienpädagogik kaum zwischen unterschiedlichen Virtualitätskonzepten unterschieden. Die Rede von «virtuellen Lernformen» oder «virtuellen Gruppen» könnte erheblich ausdifferenziert werden angesichts der diversen Auffassungsweisen von «virtuell» in der Philosophie (Bsp. Virtualität als «Emergenz-Boden» jeglicher Aktualität), der Physik (Bsp. geometrisch konstruierte, scheinbare Bilder oder «virtuelle Teilchen») und der Medientheorie (Bsp. hybride Bildformen und Formen der Modalisierung medialisierter Wirklichkeitserfahrungen). 


\begin{tabular}{|c|c|c|c|c|c|}
\hline Medien & Primärgruppe & $\begin{array}{c}\text { Ethnische } \\
\text { Gemeinschaften }\end{array}$ & Gemeinwesen & $\begin{array}{l}\text { bereichsspezifische } \\
\text { Gemeinschaften }\end{array}$ & Communities \\
\hline Kommunikationsmittel & Freundschaftsmünze & $\begin{array}{c}\text { Sprachliche } \\
\text { Besonderheiten von } \\
\text { Ethnien }\end{array}$ & Unterschriftenliste & $\begin{array}{l}\text { Braillenotenschrift - } \\
\text { Lerngruppe }\end{array}$ & Social welfare flyer \\
\hline Medienangebote & Familienalbum & Ethno-Musik & $\begin{array}{l}\text { sozialpolitisch } \\
\text { motivierte } \\
\text { Regionalsendungen }\end{array}$ & Fussball-Fanzine & $\begin{array}{c}\text { Business-Networking } \\
\text { Informationen } \\
\text { (Bsp. http://www.femity.net) }\end{array}$ \\
\hline $\begin{array}{l}\text { Instrumente, Geräte, } \\
\text { Apparate }\end{array}$ & Babyfonef & Volkmusikinstrumente & Bürgernetz-Server & Filmclub & $\begin{array}{c}\text { Fotocommunitics (Bsp. } \\
\text { http://www.fotocommunity.de/) }\end{array}$ \\
\hline Medienorganisationen & $\begin{array}{l}\text { Gemeindemusik- } \\
\text { kapelle }\end{array}$ & Ethno-Sender & Lokalradio & $\begin{array}{c}\text { Downtown Community } \\
\text { TV (NY) } \\
\text { (http://www.dctvny.org/) }\end{array}$ & $\begin{array}{l}\text { Neue Medien Vereine } \\
\text { (Bsp. http://www.lag-neue- } \\
\text { medien.de/) }\end{array}$ \\
\hline Medium / Form & Blutsverwandtschaft & $\begin{array}{c}\text { Distinktion ,zugehörig } \\
\text { / fremd“" }\end{array}$ & Nachbarschaft & Gefahrengemeinschaft & Web-Community \\
\hline $\begin{array}{l}\text { symbolisch generalisierte } \\
\text { Kommunikationsmedien }\end{array}$ & $\begin{array}{l}\text { Freundschaftliche } \\
\text { Zuwendung }\end{array}$ & $\begin{array}{l}\text { Anerkennung } \\
\text { ethnischer } \\
\text { Minderheiten }\end{array}$ & $\begin{array}{l}\text { Verteilungsmacht } \\
\text { in der } \\
\text { Gemeinwesenarbeit }\end{array}$ & $\begin{array}{l}\text { Bereichsspezifische } \\
\text { Nutzung von } \\
\text { Komplemen- } \\
\text { tärwährungen }\end{array}$ & $\begin{array}{c}\text { Payboxer } \\
\text { (http://www.payboxer.at/) }\end{array}$ \\
\hline
\end{tabular}

Abb. 1: Medien-Gemeinschaft: Ausgewählte Begriffe und diskursive Schnittstellen 
Wie lässt sich nun die Komplexität unserer Thematik in den Griff bekommen, wenn die Vielfalt medialer Formen und Medienkonstellationen sowie der intermedialen Bezüge noch komplexere Betrachtungsweisen nahe legt? In einem ersten Anlauf lassen sich durchaus einige Dimensionen benennen, die für die Charakterisierungen im Spannungsfeld von Medien und Gemeinschaften relevant sind. Wir fassen diese in der folgenden Punktation zusammen:

\section{Vertrautheit und emotionale Bindung}

Hier geht es um Abstufungen von Fremdheit, Bekanntheit und Vertrautheit sowie emotionale Aspekte und affektlogische Zusammenhänge (vgl.

Ciompi 1997).

\section{- soziale Abgrenzung und Offenheit}

Diese Dimensionen hebt ab auf Zugangsregelungen, Modalitäten der Zugehörigkeit und Grade der mehr oder weniger flexiblen oder rigiden Offenheit und Schliessung.

\section{- raum-zeitliche Begrenzung}

Räumliche und zeitliche Reichweiten eröffnen sehr unterschiedliche Basisverständnisse (kurzlebig, auf eine gewisse Zeit, mittelfristig, lebenslänglich, auf enge oder weite Erfahrungsräume bezogen, global ausgerichtet usw.)

\section{- Zweck und Profit}

Hier geht es um den Fokus der Aktivitäten, gemeinsame Aufgaben, Modalitäten der Anerkennung, Formen des Profits einschliesslich der Früchte gemeinnütziger Bemühungen.

\section{- Bindung / Bindemodalität}

Bindung kann auf sehr unterschiedliche Weisen hergestellt werden, angefangen von moralischen oder politischen Überzeugungen über technologiegetriebene Qualitäten bis zu konsumorientierten Handlungsund Verhaltensweisen.

\section{- Solidarität}

Die Relation von Gemeinschafts-, Gruppen- und Einzelinteressen kann sehr verschiedenen gestaltet sein.

\section{- Stimulation und Stabilität}

Hier geht es um statische und dynamische Sichtweisen und Selbstverständnisse sowie um Grade der Stimulationstoleranz.

\section{- Aktivität und Teilhabe}

Teilhabe durch gemeinsames Tun und Handeln, Formen der Anteil- nahme oder der Partizipation korrespondieren mit heterogenen Erfahrungen.

\section{- Faktizität und Fiktionalität}

Das Zusammenspiel fiktionaler und realer Züge im Sinne eines Sowohlals-auch kann komplexe Dynamiken und sehr unterschiedliche Qualitäten annehmen, die das gesamte Spektrum von Ehe-Gemeinschaften bis hin zu den Communities der Blogger, Modder und Podder ${ }^{5}$ betreffen.

\section{- Medialität}

Sine-qua-non-Charakter hat die Dimension der Medialität, die sich auf das explizite oder implizite Verständnis und die Rolle von Medien bezieht (Bsp. Medieninstitutionen, Medientechnologien, Medienprogramme und -formate, Form als temporale Aktualisierung eines Mediums, usw.).

Diese Dimensionen sind unseres Erachtens für unterschiedlichste Typen von Mediengemeinschaften bedeutsam. Wir können als Zwischenfazit festhalten, dass Klassengemeinschaften, Lebensgemeinschaften, Wirtschaftsgemeinschaften, Ordensgemeinschaften, Sportgemeinschaften, Lerngemeinschaften, «virtuelle Gemeinschaften», Bürgernetzwerke oder RadioCommunities durch unterschiedliche Auffassungen von Medien und Gemeinschaft konstitutiert werden und in entsprechend vielfältiger Weise anzutreffen sind. Während es unter Marketing-Gesichtspunkten primär um «trendige» und Gewinn versprechende Bezeichnungen geht, stellt sich in theoretisch motivierter Perspektive die Frage, ob wir uns mit einer Vielfalt an heterogenen und konzeptionell unverträglichen Medien-Gemeinschaften zufrieden geben müssen, oder ob sich Zusammenhänge der diversen Auffassungen auch in integrativer Weise argumentieren lassen.

Medien-Gemeinschaft: Eine Pluralität inkommensurabler Beschreibungen? Wer sich mit einer unbestimmten Pluralität inkommensurabler Beschreibungen nicht zufrieden geben will und eine umfassende Perspektive, die gleichsam alle verschiedenen Auffassungen von Medien-Gemeinschaft unter einem Dach vereinen könnte, für problematisch hält, kann sich auf pragmatische Begründungen für situations- und fallbezogener Ent-

${ }^{5}$ Die Bezeichnungen sind in Medienteilkulturen in Gebrauch und erfolgen in Anlehnung an Weblog-Betreiber/innen, Mitglieder von Mod(ification)-Cultures und Podcasting-Gruppen. 
scheidungen berufen. ${ }^{6}$ Eine theoretisch brauchbare Alternative besteht in der Interpretation der erwähnten «Dimensionen» auf dem Hintergrund des Variationen-Konzepts von Nelson Goodman und Catherine Z. Elgin (vgl. Goodman / Elgin 1989, Goodman 1995). Das Ziel besteht dabei in der Relationierung der Beschreibungen der unterschiedlichen Phänomenbereiche als Variationen über ein Thema.

Fragen nach der Relation von Begründungsansätzen und der Pluralität von Perspektiven spielen in der Philosophie seit Jahrhunderten eine Rolle. Mit dem Aufkommen der Postmoderne-Diskurse allerdings wurden Probleme der Heterogenität, Differenz und Diversität zugespitzt wie nie zuvor. Möglichkeiten der Konstruktion, Kombination und Rekombination von Erkenntnisfragmenten wurden sehr unterschiedlich gefasst. Einer der prominentesten und vielseitigsten Lösungsansätze, die im Kontext konstruktivistischer Diskurse entfaltet worden sind, ist in den «Weisen der Welterzeugung» (Goodman 1995) angelegt. Wir wollen zur Beschreibung und Analyse der Gemeinsamkeiten und Unterschiede von Mediengemeinschaften auf einige seiner Überlegungen zurückgreifen.

Wenn wir davon ausgehen, dass wir es in vielerlei Hinsicht mit verschiedenen Welten zu tun haben, dann stellt sich die Frage nach einer «neutralen», zugrunde liegenden Welt. Goodman schreibt dazu:

«Der Physiker hält seine Welt für die reale, indem er Tilgungen, Ergänzungen, Unregelmässigkeiten und Betonungen anderer Versionen der Unvollkommenheit der Wahrnehmungen, den Dringlichkeiten der Praxis oder der dichterischen Freiheit zuschreibt. Der Phänomenalist betrachtet die Wahrnehmungswelt als fundamental, die Beschneidungen, Abstraktionen, Vereinfachungen und Verzerrungen anderer Versionen hingegen als Ergebnis von wissenschaftlichen, praktischen oder künstlerischen Interessen. Für den Mann auf der Strasse weichen die meisten Versionen der Wissenschaft, der Kunst und der Wahrnehmungen auf mancherlei Weise von der vertrauten und dienstbaren Welt ab, die er aus Fragmenten der wissenschaftlichen und künstlerischen Überlieferung und aus seinem eigenen Überlebenskampf zusammengebastelt hat. Am häufigsten wird in der Tat diese Welt für real gehalten; denn Realität in einer Welt ist - wie der Realismus eines Bildes - grösstenteils eine Sache der Gewohnheit.

${ }^{6}$ Unabhängig davon, ob dabei einfache oder komplexe Begründungsmodelle (vgl. Sieckenius de Souza / Preece 2004) verwendet werden, ist damit die theoretische Frage nach der Relation der verschiedenen Konzepte von Medien-Gemeinschaften meistens weder thematisiert noch beantwortet.
Ironischerweise wird also unsere Leidenschaft für eine Welt $\mathrm{zu}$ verschiedenen Zeiten und für verschiedene Zwecke auf viele verschiedene Weisen befriedigt [Hervorhebung i.Org.]» (Goodman 1995, 34 f).

Weder die Welt der Physik, noch die der Phänomenologie oder die des Alltagslebens kann ohne weiteres als eine 〈Einheit〉 betrachtet werden. Das hat nichts mit einem Erkenntnismangel zu tun. Wir kommen in vielen Bereichen immer wieder in die Lage entscheiden zu müssen, ohne ausreichend über relevante Informationen und finanzielle oder zeitliche Ressourcen zu verfügen. Es geht also nicht um die Mangelhaftigkeit und Unvollständigkeit der Erkenntnisse, auf die man sich bei diesen Entscheidungen stützen muss. Vielmehr geht Goodman als Konstruktivist davon aus, dass die Welt vielmehr Produkt statt Input der Beschreibung ist. Weil viele Möglichkeiten der Beschreibung in Betracht kommen, gibt es nach Goodman ebenso viele Welten. Jeder Ausgangspunkt und jeder Gesichtspunkt führt in der Beschreibung zu spezifischen Fakten, den Fakten der jeweiligen Welt. Dies lässt sich beispielsweise anhand der Frage nach der Erdbewegung verdeutlichen. Wenn man davon ausgeht, dass die Erde stillsteht, dann resultiert daraus das Faktum, dass die Sonne sich bewegt und umgekehrt.

«Die Erde, die wahrheitsgetreu als bewegt beschrieben wird, ist nicht die Erde, die wahrheitsgetreu als stillstehend beschrieben wird. Und die Welt der einen hat für einen Planeten wie der andere keinen Platz. Wenn also beide Beschreibungen wahr sind, sind sie in verschiedenen Welten wahr.

Dann gibt es, wenn überhaupt, viele Welten» (Goodman / Elgin 1989, 73). Eine Bewegung der Erde oder der Sonne <an sich〉 können wir - zumindest ohne die Einführung neuer konzeptioneller Rahmen - nicht feststellen. Es handelt sich hier offensichtlich um konfligierende Aussagen. Und solche sind Goodman und Elgin (ebd., 72) zufolge - wenn überhaupt - in unterschiedlichen Welten wahr. Wir können jeweils unterschiedliche Perspektiven einnehmen, wir können diese aber nicht in einer umfassenden Perspektive zusammenführen oder gleichsam von einem übergeordneten, <umfassenden〉 Beobachtungs-Standpunkt aus allgemeingültig beurteilen. Diese Welten werden erzeugt,

«indem man mittels Wörtern, Zahlen, Bildern, Klängen oder irgendwelchen anderen Symbolen in irgendeinem Medium solche Versionen erzeugt; und die vergleichende Untersuchung dieser Versionen und Sichtweisen sowie ihrer Erzeugung ist das, was ich eine Kritik der Welterzeugung nenne» (Goodman 1995, 117). 
Die Fragmentierung der Erkenntnis und die Vielheit von Welten stellt auch im Kontext von Medien und Gemeinschaften eine Schwierigkeit dar. Anstelle einer Auflistung möglicher Definitionen schlagen wir vor, die erwähnten Dimensionen als Gesichtspunkte aufzufassen, aus denen verschiedene Beschreibungen von Medien und Gemeinschaft jeweils als Variationen über ein Thema zu (re)konstruieren sind.

Der Variationsbegriff, um den es hier geht, bezieht sich nicht auf die Psychologie oder Soziologie der Welterzeugung, sondern auf philosophische Aspekte der wechselseitigen Beziehungsmöglichkeiten unterschiedlicher Welten. Dabei können Variationen prinzipiell als Repräsentationen eines Originals verstanden werden. Das Original 〈an sich〉 - in unserem Fall wäre das eine Beschreibung einer Art «UrMediengemeinschaft» - kann aber unmöglich als Kriterium zum Vergleich der variierenden Beschreibungen mit dem Original dienen. Vielmehr werden die Ähnlichkeiten der Beschreibungsvarianten erst vermittels der Perspektiven hervorgebracht, die zugleich auch einen Unterschied zwischen Variation und Original erzeugen (vgl. Goodman / Elgin 1989, 93 ff). ${ }^{7}$ Die Ähnlichkeiten mit dem Original, die Variationen zu Variationen machen, werden jeweils von einer bestimmten Perspektive erst erzeugt. Dabei spielen metaphorische Übertragungsleistungen und die Differenzierung gemeinsamer und variierender konzeptueller Dimensionen eine entscheidende Rolle. Wie bei der Metapher geht es bei der Variation um Ähnlichkeit und Kontrast zugleich. Nebst dieser formalen Bedingung ist eine funktionale erforderlich. Goodman und Elgin erläutern dies am Beispiel der Musik:

«... um als eine Variation zu funktionieren, muss eine in Frage kommende Passage die erforderlichen gemeinsamen Merkmale des Themas buchstäblich und die erforderlichen kontrastierenden Merkmale des Themas metaphorisch exemplifizieren und auf es über diese Merkmale Bezug nehmen. Eine Variation zu sein beruht darauf, dass sie als solche funktioniert ...» (Goodman / Elgin 1989, 100).

So ist auch eine Variation nicht 〈an sich〉 eine Variation, sie funktioniert vielmehr als solche. Wenn es also nicht um einen Vergleich diverser Beschreibungen von Mediengemeinschaften mit einer «Urbeschreibung» geht, wie kann dann der Ansatz von Goodman und Elgin hier fruchtbar

\footnotetext{
Am Beispiel der Meninas-Variationen von Picasso zeigen sie, dass nicht das Original des Velázquez' selbst Picassos Variationen erklären kann, sondern eine spezifische Perspektive, aus der Original und Variationen erst als solche erscheinen (ebd., 93 ff). $21 / 29$

gemacht werden? Dies ist möglich, wenn wir (1) die konzeptionelle Ebene ins Blickfeld rücken und uns (2) auf eine vergleichende Untersuchung verschiedener Versionen anhand konstrastierender Merkmale konzentrieren. Zur Verdeutlichung greifen wir jeweils exemplarisch drei Konzeptionen (B. Anderson, A. Etzioni, S. Lash) und drei Gesichtspunkte (Bindung, Medialität, Faktizität / Fiktionalität) heraus, aus denen verschiedene Beschreibungen von Medien und Gemeinschaft jeweils als Variationen über ein Thema rekonstruiert werden können (s. Abb. 2).

Der erste Gesichtspunkt, aus dem die drei Beschreibungen als Variationen über ein Thema zu (re-)konstruieren sind, ist mit dem Merkmal Bindung verknüpft. Dieses Merkmal macht die Ansätze der drei Autoren zu Variationen von Mediengemeinschaften. Zugleich kommen aus dieser Perspektive folgende Kontraste ans Licht:

- die herrschaftsförmige Inszenierung der Bindung im Konzept von B. Anderson,

- der Stellenwert von Pflicht und sozialer Verantwortung bei A. Etzioni,

- die eigene Wahl der Bindungen bei S. Lash im Lichte ethischer Überlegungen.

Ein zweiter möglicher Focus definiert Mediengemeinschaft über das Merkmal Medialität, das für alle drei Konzepte konstitutiv ist. Die Verschiedenheit resultiert in dieser Perspektive aus den jeweiligen Medienverständnissen und deren Stellenwert. Dieser besteht

- in der Bedeutung der «Heilige Sprache» sowie der nationalen Rundfunkanstalten bei B. Anderson,

- in der Kritik der Medienkonzentration und der Favorisierung von Community Media bei A. Etzioni,

- in der Bedeutung medienästhetischer Potenziale bei S. Lash.

Auch die dritte Perspektive bringt ihre eigene Kontrastierung der drei jetzt über das Merkmal Faktizität / Fiktionalität definierten - Varianten von Mediengemeinschaften mit sich. Sie besteht

- in der generellen Charakterisierung von Gemeinschaften als «vorgestellte Gemeinschaften» bei B. Anderson,

- in der Reaktivierung von Bürgergesellschaften angesichts bedrohter Demokratien im Spannungsfeld faktischer Entwicklungen und fiktionaler Vorstellungen bei A. Etzioni,

- im Bewusstsein der Konstruiertheit der Gemeinschaften bei S. Lash. 
In allen drei Konzeptionen kommen sowohl faktische als auch fiktionale Züge - allerdings in sehr unterschiedlichen Weisen - zum Tragen.

\begin{tabular}{|l|c|c|c|}
\hline Kimension & B. Anderson & A. Etzioni & S. Lash \\
\hline Bindung & $\begin{array}{c}\text { herrschaftsförmige } \\
\text { Inszenierung }\end{array}$ & $\begin{array}{c}\text { Pflicht, soziale } \\
\text { Verantwortung }\end{array}$ & $\begin{array}{c}\text { selbst gewählt, } \\
\text { Ethik basiert }\end{array}$ \\
\hline Medialität & $\begin{array}{c}\text { «Heilige Sprache», } \\
\text { nationale Rundfunk- } \\
\text { anstalten }\end{array}$ & $\begin{array}{c}\text { Community Media, } \\
\text { Kritik der Medien- } \\
\text { konzentration }\end{array}$ & Medienästhetik \\
\hline Faktizität / Fiktionalität & $\begin{array}{c}\text { kvorgestellte } \\
\text { bemeinschaft» } \\
\text { Demokratie, } \\
\text { Reaktivierung von } \\
\text { Bürgergesellschaft }\end{array}$ & $\begin{array}{c}\text { Bewusstsein der } \\
\text { Konstruiertheit }\end{array}$ \\
\hline
\end{tabular}

Abb. 2: Medien - Gemeinschaft: exemplarische Anwendung einzelner Gesichtspunkte I

Analog liessen sich auch die anderen genannten Merkmale in kontrastiver Weise vergleichend untersuchen. Dies ist nicht nur im Hinblick auf theoretische Konzeptionen, sondern auch im Zusammenhang praktisch ausgerichteter Konzepte möglich. Zwecks Verdeutlichung greifen wir wiederum drei Beispiele heraus, mit denen wir u. a. im Frühjahr 2004 im Rahmen der Tagung «Media Communties» (s. http://www.mediacommunities.net/) befasst waren:

- Association of Independents in Radio (AIR):

http://www.airmedia.org/

- LizzyNet - die Community für Mädchen und junge Frauen von Schulen ans Netz e. V.: http://www.lizzynet.de/

- Global Radiopharmacy Community (VirRAD):

http://community.virrad.eu.org/

Ähnlich wie im vorigen Fall lassen sich auch bei diesen Mediengemeinschafts-Konzepten anhand der ausgewählten Merkmale Ähnlichkeiten und kontrastierende Elemente herausarbeiten (vgl. Abb. 3).

Das Merkmal Bindung lässt sich beispielsweise kontrastieren, indem die entsprechend unterschiedlichen Akzentuierungen («Bindemittel») rekonstruiert werden. So stehen im Vordergrund
- politische Ansprüche und ähnlich gelagerte Interessenslagen der Mitglieder bei AIR,

- Geschlechter differenzierende und Peergroup-Überlegungen sowie die Möglichkeit der Moderation für fortgeschrittene Mitglieder und die intensive Nutzung eines Treffpunkts bei LizzyNet,

- die Idee eines technologie- und bildungsgetriebenen Ansatzes («self learning for work») bei VirRAD.

Im Hinblick auf das Merkmal Medialität lassen sich ebenfalls ähnliche, im Detail jedoch durchaus unterscheidbare Verständnisse ausmachen. Diese bestehen

- im Zusammenspiel von Content Management System (CMS), Newsletter, Lernumgebung und Radioproduktionen bei AIR,

- in der Typik der dreiteiligen Lern- und Kommunikationsplattform (Magazin, Community, Know How) bei LizzyNet,

- im Einsatz multimedialer Course-ware, des 3D Laboratory und den Kommunikationswerkzeugen bei VirRAD.

Last but not least bietet auch die dritte Perspektive Optionen der Kontrastierung anhand des Merkmals Faktizität / Fiktionalität. Sie bestehen grosso modo

- in der Dynamik von Kooperation und Konkurrenz sowie der heterogenen Mitgliedschaften in punkto Alter und geografische Verteilung bei AIR,

- in der Spannung prinzipieller Offenheit für Mädchen aller Milieus und Herkünfte und der faktischen Nutzung durch Teilgruppen (überwiegend Gymnasiastinnen) bei LizzyNet,

- in den Grenzen der Planbarkeit bzw. «Herstellung» der Gemeinschaft und dem faktischen «link up» einer bestehenden Community bei VirRAD. 


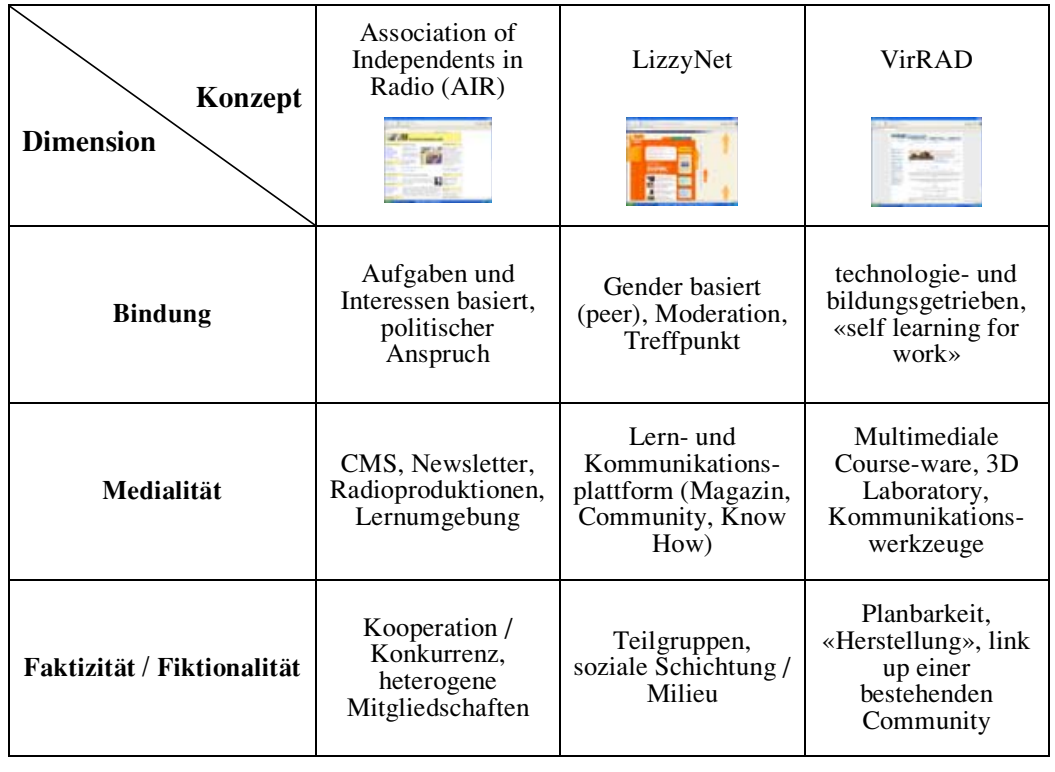

Abb. 3: Medien-Gemeinschaft: exemplarische Anwendung einzelner Gesichtspunkte II

Alle angesprochenen Charakterisierungen von Mediengemeinschaften lassen sich damit auf unterschiedliche Weisen als Variationen eines Phänomens beschreiben, ohne dass eine ontologische Festlegung erforderlich wäre. Sie sind vielmehr Entwürfe unterschiedlich medialisierter Gemeinschaftsformen, deren Gemeinsamkeiten und kontrastierende Besonderheiten gleichsam durch Modulationen hervorgerufen werden. Goodmans Variationsbegriff erweist sich damit auch im Kontext gemeinschaftsbezogener «Weisen der Welterzeugung» (Goodman 1995) als fruchtbares Analyseinstrument, das eine relationale Verbindung der verschiedenen Perspektiven ohne Auflösung derselben in eine diffuse Vielheit von Konzepten und ohne Rekurs auf eine praktisch, theoretisch oder ideologisch fixierte Vorstellung von Mediengemeinschaft ermöglicht.

\section{Medien-Gemeinschaft: Resümee}

Was bringen nun diese Überlegungen zum Themenbereich Mediengemeinschaften im Allgemeinen und für die medienpädagogische Theorie und Praxis im Besonderen? Wir hoffen, dass sie dazu beitragen, uns für die komplexen sozialen und kulturellen Fragen zu sensibilisieren, denen wir uns alle unter den gegenwärtigen Bedingungen stellen müssen: Wo sehen wir unseren Platz? Wo fühlen wir uns zugehörig? Und wir sind gefordert, sowohl die Rolle der Medien in den Antworten auf diese Fragen kritisch zu hinterfragen als auch das Potenzial, das sich hier im Hinblick auf ein gleichwertiges menschliches Zusammenleben und die Ermächtigung der Einzelnen auftut, nutzbar zu machen.

Wir hoffen weiters, dass die Überlegungen zu differenzierten Betrachtungsweisen und insbesondere zur Weiterentwicklung von Konzepten «virtueller (Lern-)Gemeinschaften» (s. z. B. Döring 2001, Seufert 2002) anregen. Die Medienpädagogik kann hier jenseits aller Buzzword-Inszenierungen wichtige Beiträge leisten. Ihr Potenzial zur Vermeidung von «educational lags» ${ }^{8}$ und zur zukunftsorientierten Gestaltung pädagogischer Reformprozesse (Hug 2005) ist höher denn je. Gerade im Spannungsfeld von Mediendidaktik und Mediensozialisationsforschung kommen jene Prozesse der Medialisierung zum Tragen, die neue Formen der Vergemeinschaftung mit sich bringen und die auf meta-theoretischer, konzeptioneller und auf praktischer Ebene bearbeitet werden müssen. Reduktionismus und allzu oberflächliche Zugänge werden kaum weiterhelfen. Entsprechend lautet ein weiteres Resümee - frei nach Nelson Goodman: «There are many media communities, if any!»

\section{Literaturverzeichnis}

Anderson, Benedict. Die Erfindung der Nation - zur Karriere eines erfolgreichen Konzepts. Frankfurt/Main: Campus, 1988.

Bauman, Zygmunt. Community. Seeking Safety in an Insecure World. Cambridge/Oxford/Malden: Polity Press, 2001.

Beck, Ulrich. Risikogesellschaft - auf dem Weg in eine andere Moderne. Frankfurt/Main: Suhrkamp, 1995.

Beck, Ulrich. «Das Zeitalter der Nebenfolgen und die Politisierung der Moderne.» In: Reflexive Modernisierung. Eine Kontroverse. Hrsg. v. Ulrich Beck, Anthony Giddens u. Scott Lash. Frankfurt/Main: Suhrkamp, 1996. S. 19-112.

${ }^{8}$ Unter «educational lags» verstehen wir in Abwandlung von William F. Ogburn's (1950) Theorie des sozialen Wandels die verzögerten Entwicklungsdynamiken pädagogischer und bildungspolitischer Bereiche in Relation zu technologischen Bereichen. 
Billig, Michael. Banal Nationalism. London/Thousand Oaks/New Delhi: Sage, 1995

Ciompi, Luc: Die emotionalen Grundlagen des Denkens. Entwurf einer fraktalen Affektlogik. Göttingen: Vandenhoeck \& Ruprecht, 1997.

Corner, John. «Television and Culture: Duties and Pleasures». In: British Cultural Studies. Geography, Nationality, and Identity. Hrsg. v. David Morley u. Kevin Robins. Oxford: Oxford University Press, 2001. S. 261-272.

Döring, Nicola. «Virtuelle Gemeinschaften als Lerngemeinschaften!? Zwischen Utopie und Dystopie.» Zeitschrift für Erwachsenenbildung. Dokument aus dem Internetservice Texte online des Deutschen Instituts für Erwachsenenbildung, 2001.

〈http://www.die-frankfurt.de/zeitschrift/32001/positionen4.htm〉 (12.03.2005).

Etzioni, Amitai. «Introduction». In: The Essential Communitarian Reader. Hrsg. v. Amitai Etzioni. Lanham/Boulder/New York/Oxford: Rowman \& Littlefield, 1998. S. IV-XXIV.

Giddens, Anthony. «Leben in einer posttraditionellen Gesellschaft.» In: Reflexive Modernisierung. Eine Kontroverse. Hrsg. v. Ulrich Beck, Anthony Giddens u. Scott Lash. Frankfurt/Main: Suhrkamp, 1996. S. 113-194.

Giddens, Anthony. Enfesselte Welt. Wie die Globalisierung unser Leben verändert. Frankfurt/Main: Suhrkamp, 2001.

Goodman, Nelson / Elgin, Catherine Z.: Revisionen: Philosophie und andere Künste und Wissenschaften. Frankfurt/M.: Suhrkamp, 1989 (engl. Org. 1988)

Goodman, Nelson: Weisen der Welterzeugung. Frankfurt/M.: Suhrkamp, ${ }^{3} 1995$ (engl. Org. 1978).

Hall, Stuart. Rassismus und kulturelle Identität. Ausgewählte Schriften 2. Hamburg: Argument-Verlag, 1994.

Hug, Theo. «Beiträge der Medienpädagogik zur Vermeidung von «educational lags». Ein Plädoyer für zukunftsorientierte Reformen unter den Auspizien des «mediatic turn».» In: Medienimpulse, 13. Jg. / Heft 53, 2005 (im Erscheinen).

Innis, Harold A. The Bias of Communication. Toronto: Toronto University Press, 1951.

Innis, Harold A. Empire and Communications. Toronto: Toronto University Press, 1972.
Lash, Scott. «Reflexivität und ihr Doppelungen: Struktur, Ästhetik und Gemeinschaft.» In: Reflexive Modernisierung. Eine Kontroverse. Hrsg. v. Ulrich Beck, Anthony Giddens u. Scott Lash. Frankfurt/Main: Suhrkamp, 1996. S. 195-286.

Lévy, Pierre. Collective Intelligence. Mankind's Emerging World in Cyberspace. Cambridge, Mass.: Perseus Books, 1997.

Luhmann, Niklas: Soziale Systeme. Frankfurt/M.: Suhrkamp, 1987.

Luhmann, Niklas: Die Kunst der Gesellschaft. Frankfurt/M.: Suhrkamp, 1995.

Morley, David. «Nicht 〈zu Hause〉 in der Mediennation.» In. Bewegte Identitäten. Medien in transkulturellen Kontexten. Hrsg. v. Brigitta Busch, Brigitte Hipfl u. Kevin Robins. Klagenfurt: Drava, 2001. S. 2146.

Ogburn, William F.. Social change with respect to culture and original nature. New York: Viking Press. 1950 (first published in 1922).

Rheingold, Howard. Virtuelle Gemeinschaft. Soziale Beziehungen im Zeitalter des Computers. Bonn: Addison-Wesley, 1994.

Robins, Kevin/Aksoy, Asu. «〈Abschied von Phantomen»: Transnationalismus am Beispiel des türkischen Fernsehens.» In: Bewegte Identitäten. Medien in transkulturellen Kontexten. Hrsg. v. Brigitta Busch, Brigitte Hipfl u. Kevin Robins. Klagenfurt: Drava, 2001. S. 71-110.

Schmidt, Siegfried J. (Hg.): Kognitive Autonomie und soziale Orientierung. Konstruktivistische Bemerkungen zum Zusammenhang von Kognition, Kommunikation, Medien und Kultur. Frankfurt/M.: Suhrkamp, 1994.

Seufert, Sabine. «Virtuelle Lerngemeinschaften: Konzepte und Potenziale für die Aus- und Weiterbildung.» In: Ergebnisbericht des Bundesinstituts für Berufsbildung, 2002.

〈http://www.scil.ch/seufert/docs/virtuelle-lerngemeinschaften.pdf〉 (12.03.2005).

Shaviro, Steven. Connected, or What it Means to Live in the Network Society. Minneapolis/London: University of Minnesota Press, 2003.

Sieckenius de Souza, Clarisse / Preece, Jenny. «A framework for analyzing and understanding online communities.» In: Interacting with Computers. The Interdisciplinary Journal of Human-Computer Interaction. Volume 16, Issue 3, June 2004. 579-610.

Tönnis, Ferdinand. Gemeinschaft und Gesellschaft. Grundbegriffe der reinen Soziologie. Darmstadt: Wissenschaftliche Buchgesellschaft, 1972. 
Web-Adressen (Stand: jeweils 12.03.2005)

http://community.virrad.eu.org/>

〈http://de.wikipedia.org/wiki/Apostel_Paulus〉

〈http://www.airmedia.org/>

http://www.dctvny.org/>

〈http://www.die-frankfurt.de/zeitschrift/32001/positionen4.htm

〈http://www.femity.net/>

〈http://www.fotocommunity.de/>

〈http://www.lag-neue-medien.de/

〈http://www.live365.com/stations/dimmsummer/>

http://www.lizzynet.de/>

http://www.media-communities.net/>

〈http://www.payboxer.at

〈http://www.scil.ch/seufert/docs/virtuelle-lerngemeinschaften.pdf〉

\section{Filme}

Gegen die Wand, 2003, Regie: Fatih Akin

Bend it like Beckam, 2002, Regie: Gurinder Chadha 